\title{
BIM in teaching - lessons learned from exploratory study
}

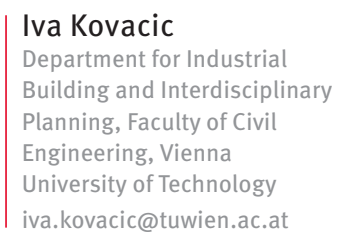

Rüdiger Suppin

Department for Industrial

Building and Interdisciplinary

Planning, Faculty of Civil

Engineering, Vienna University

of Technology

rsuppin@industriebau.tuwien.ac.at

\author{
Dragos Vasilescu \\ Institute for Management \\ Sciences, Faculty for Mecha- \\ nical Engineering, Vienna \\ University of Technology \\ dragos.vasilesc@ \\ tuwien.ac.at
}

\author{
Lars Oberwinter \\ Department for Industrial \\ Building and Interdisciplinary \\ Planning, Faculty of Civil \\ Engineering, Vienna \\ University of Technology \\ lars.oberwinter@tuwien.ac.at
}

Michael Filzmoser

Institute for Management

Sciences, Faculty for Mechanical Engineering, Vienna University of Technology michael.flizmoser

@tuwien.ac.at
DOI 10.5592/otmcj.2015.3.3 Research paper

\section{Keywords}

BIM; Exploratory study; Colaboration; Simulation; Analysis
BUILDING INFORMATION TECHNOLOGY BEARS PROMISE TO BRING INTEGRATION INTO FRAGMENTED AEC INDUSTRY, AS WELL AS LARGE POTENTIALS FOR OPTIMIZATION AND MANAGEMENT OF BUILDING PERFORMANCE ALONG LIFE CYCLE. However, the adoption in Central Europe is much slower than in the USA or Scandinavia; the designers and planners are sceptical about BIM benefits. In order to build up capabilities and thus support BIM adoption in the practice, BIM skills have be built up already in university teaching. This endeavour is the central aim of the BIM_sustain project accomplished at the Vienna University of Technology. In winter term 2012/13 and winter term 2013/14 we accomplished interdisciplinary BIM-supported design labs with student participants from architecture, civil engineering and building science. The teams used different modelling and simulation software constellations for building design and analysis. The software-constellations were evaluated in terms of BIMinteroperability, and the design process was documented by means of time and activity assessment, surveys on team performance, process satisfaction and technology acceptance and focus group interviews. In this paper we will present the results of the evaluation of both courses and analyse the differences resulting from the different course design in the two consequent terms. The first course was dominated by the issue of interfaces, whereas the second course, where better functioning software combinations in terms of data transfer were used, was dominated by the issues related to the collaboration and teamwork. Our results are not only informative for the configuration of interdisciplinary BIM-supported university teaching, but can be derived for the practice as well, especially in the areas of project management, software usage, modelling conventions or incentive systems. 


\section{INTRODUCTION}

Building Information Modelling (BIM) as powerful tool bears significant potential to enhance the integrated project delivery, as well as to accelerate its implementation in the practice (Fellows and Liu, 2010).Thereby $B I M$ represents a catalyst for the reduction of largely fragmented AEC (CWIC, 2004) - so is the hope both on the academic as well as industry side. BIM tools offer support for interdisciplinary planning and design, where by digital, parametric building model represents a joint knowledge base, enabling interdisciplinary data exchange and management along the life cycle of a building (Penttilä, 2006). BIM tools offer numerous possibilities for coupling of various analysis and simulation tools, thus enabling energy and resources analysis and optimization in the early design stages (Azhar et al., 2011).

The adoption of BIM tools is significantly slower in Central Europe compared to the Scandinavian or AngloSaxon countries (McGraw-Hill, 2010). Further on, empirical evidences for actual BIM benefits are hardly available (Jung and Joo, 2011), since very few of the adopters are actually employing any measuring strategies. The greatest challenges for the implementation of BIM tools have been identified outside of the technical domain - in the realm of processes, stakeholdernetworks and policies (Gu and London, 2010; Linderoth, 2010; Succar, 2009).

Within this background, a research project BIM_sustain funded by Austrian Funding Agency FFG was carried out at the Vienna University of Technology, as research led teaching, in order to analyse the benefits of BIM supported planning and to develop a framework for guidelines for the AEC practice. The project was carried out in the cooperation of three Departments of the Faculties for Civil Engineering, Architecture and Mechanical Engineering, together with seven BIM software developers and vendors. The research aim was to identify BIM potentials for process-integration; through empirical research using experimental study. The study was carried out as interdisciplinary design course, taking place in the winter semester of 2012/13 (pilot experiment) and 2013/14; with follow up in $2014 / 15$, with the students of architecture, civil engineering and master of building science.

This paper is structured as following: In the next chapter we will present the design and the structure of the empirical study (courses), the team-structure and used softwareconstellations. In the third chapter we will present the results of the study obtained through quantitative analysis of the post-questionnaires, time-sheets and protocols; closer attention will be paid to the differences of the both courses resulting from the changed design of the study. In the fourth chapter we will summarise and discuss the results, presenting the experience of the teaching staff and in the last chapter we will draw the conclusions for the practice as well as for the future design of BIM-teaching classes.

\section{Experiment Design}

In order to evaluate the possible benefits of BIM supported design, we organised an experimental study as interdisciplinary, interfaculty design course: "Interdisciplinary BIMsupported planning concepts". The course was organized and carried out and as collaboration of two faculties Faculty for Civil Engineering, Institute for interdisciplinary building process management, and Faculty for Architecture, Department for Building Science and Department for Design and Architecture. The course, simultaneously the experimental study, was accompanied and evaluated by the research partner Institute for Management Sciences, Faculty for Mechani- cal Engineering, who was mainly in charge for the data gathering.

Thereby not only the students of architecture, engineering and building science were obliged to work in interdisciplinary teams, but the teaching team as well.

The course had two major aims:

$>$ To teach and demonstrate functionalities of BIM tools

> To evaluate BIM tools in interdisciplinary design setting, with special focus on interfaces

The interdisciplinary teams had an assignment to compile in collaborative manner the architectural, structural, ventilation model and conduct thermal and lightning simulation, as well as calculate the energy certificate. The models and related data had to be exchanged between the team-members. The teams worked with different software constellations, employing following software: Archicad, Allplan, Revit, Tekla, RFEM Dlubal, Scia, Plancal, Archiphysik, Dialux, Energy Plus; in order to test the compatibility and functioning of interfaces. The process was accompanied by the Institute for Management Sciences, gathering data through prequestionnaires, evaluation-questionnaires, and focus-group interviews. The students were asked to record the time-effort and conducted tasks using time sheets and protocols, which enabled exact assignment of time to specific task.

The research aim was to evaluate the multi-disciplinary collaboration and potential for enhancement of integration using BIM tools on two levels - assessing the satisfaction with technology - through evaluation of interoperability, usability and usefulness (TAM model); and satisfaction on people-process level - evaluating cooperation, results and process.

Through conducted research the insights in the functionality of BIM tools for the modelling requirements of specific disciplines (architectural 


\begin{tabular}{|c|c|c|c|c|c|c|c|c|}
\hline & & Architecture & & \multicolumn{2}{|c|}{ Civil Engineering } & & \multicolumn{2}{|c|}{ Building Science } \\
\hline & & CAD & & CAD & FEM & & CAD & Analysis \\
\hline 1 & 1 & Allplan & 1 & Allplan & Scia Engineer & 1 & Allplan & Allplan \\
\hline 2 & 1 & Revit Architecture & 1 & Revit Structure & Sofistik & 2 & Revit & Plancal \\
\hline 3 & 2 & Archicad & 1 & Tekla & Diubal & 1 & Plancal & Plancal \\
\hline 4 & 2 & Archicad & 1 & Allplan & Diubal & 2 & Plancal & Plancal \\
\hline 5 & 1 & Revit Architecture & 1 & Allplan & Scia Engineer & 1 & Plancal & Plancal \\
\hline 6 & 1 & Archicad & 1 & Allplan & Diubal & 2 & Revit & Plancal \\
\hline 7 & & Allplan & 1 & Tekla & Sofistik & 1 & Revit & Plancal \\
\hline 8 & 1 & Revit Architecture & 1 & Tekla & Scia Engineer & 1 & Allplan & Allplan \\
\hline 9 & 1 & Archicad & 1 & Revit Structure & Diubal & 1 & Plancal & Plancal \\
\hline 12 & 1 & Archicad & 1 & Allplan / Tekla & Diubal & 1 & Revit & Plancal \\
\hline 13 & 2 & Archicad & 1 & Tekla & Sofistik & 2 & Revit & Plancal \\
\hline
\end{tabular}

Figure 1: Software-Matrix and Teams in WS 2012/13

design, structural analysis, thermal and daylight simulation) as well as interoperability for data exchange between the part-taking disciplines could be assessed.

Through out the first cycle (WS 12/13) 11 teams, with overall of 35 students were taking part - including 9 architects, 11 civil engineers and 15 building scientists. In the second cycle (WS 13/14) 12 teams took part, with overall of 44 students -13 architects, 8 civil engineers, and 23 building scientists.

The assignment of the first cycle comprised a design of a low energy office building, where as the students were given a functional and spatial program, a layer structure, the preset zero, and a colour scheme for room stamps. The students were assigned to the specific software, according to their experience level, which was assessed via pre-questionnaire. The questionnaire assessed: demographic data, relevant professional experience (full employment equivalent months) as well as the experience with the single software tools.

Each team was assigned a specific software constellation, for the compilation of architectural, structural and ventilation model. The softwarematrix, reflecting simultaneously the team structure, was developed by the research (teaching) team, in order to obtain the greatest possible diversity of the software constellations (Fig. 1).

The task of the team was to develop an integrated design, consisting of architectural model comprising functional and formal concept, a load bearing structure model, ventilation model, and an energy concept with proof-of-concept (simulation and optimization) in collaborative manner.
In the following cycle (WS 13/14) the design of the course was changed based on the experience and student feedback of the former cycle. In this term the task was changed to a design of a multi-functional culture centre, providing a specific location (Margaretengürtel, 1050 Vienna). We organized a moderated Kick-off Workshop, where students were able to choose the team as well as the software. Some of the participants opted for learning the new software, choosing the team with similar interests rather than the familiar software. However, the post-questionnaire indicated that the familiar software was more important for the team-choice than choosing the team with similar interests for the most of the participants (Fig. 2).

In this term, next to the models required in the pilot-experiment (architecture, structure, ventilation and 


\begin{tabular}{|c|c|c|c|c|c|c|c|c|}
\hline & & Architecture & & \multicolumn{2}{|c|}{ Civil Engineering } & & \multicolumn{2}{|c|}{ Building Science } \\
\hline & & CAD & & CAD & FEM & & CAD & Analysis \\
\hline 1 & 1 & Allplan & 1 & Allplan & Scia Engineer & 2 & Allplan & Allplan \\
\hline 2 & 1 & Archicad & 1 & Tekla & Diubal & 2 & Plancal & Plancal \\
\hline 3 & 1 & Revit Architecture & 1 & Revit Structure & Diubal & 2 & Revit & Plancal \\
\hline 4 & 2 & Archicad & 1 & Revit Structure & Diubal & 2 & Plancal & Plancal \\
\hline 5 & 1 & Archicad & 1 & Revit Structure & Scia Engineer & 2 & Revit & Allplan \\
\hline 6 & 1 & Revit Architecture & 1 & Allplan & Scia Engineer & 2 & Plancal & Plancal \\
\hline 7 & 1 & Archicad & 1 & Allplan & Diubal & 2 & Allplan & Plancal \\
\hline 8 & 1 & Revit Architecture & 1 & Tekla & Diubal & 2 & Plancal & Plancal \\
\hline 9 & 1 & Archicad & & & & 2 & Plancal & Plancal \\
\hline 10 & 1 & Archicad & & & & 2 & Plancal & Allplan \\
\hline 11 & 2 & Archicad & & & & 2 & Revit & Allplan \\
\hline 12 & 1 & Archicad & & & & 2 & Revit & Plancal \\
\hline
\end{tabular}

Figure 2: Software-Matrix and Teams in WS 2013/14

daylight) also an acoustic simulation was a part of the assignment.

The course was in both terms accompanied by the software-companies, who offered training for specific software, according to the pre-determined schedule. The companies also supported the data transfer process and carried out the model integrity and collision proofs.

\section{Results}

The conducted study was evaluated through quantitative analysis, based on the evaluation of the post-questionnaires and of the time-sheets and protocols compiled by the students.

The post-questionnaires included inquiries related to the satisfaction with the process, result and teamwork; as well as the technology-related questions(Technology Adoption Model - TAM) including satisfaction with interoperability, usefulness and usability.

These latent constructs where measured by multiple items on a 5 -point Likert scale ranging from low/disagree (1) to high/agree (5).

After the examination of the quality of scale using Cronbachs a, the median per construct was built and the evaluation according to the discipline: Architecture (ARCH), Civil Engineering (CI) and Master of Building Science (BS); as well as over all disciplines.

In the second cycle the students were obliged to lead protocols and exact time-sheets with attributed activities, in order to enable the assessment of time-effort necessary for a specific activity.

Additionally, a qualitative evaluation was carried out in form of focusgroup interviews, also serving for debriefing of the students, where the interviews with participants of each discipline (architects, engineers and building scientists) were carried out at the end of the experiment.

The post-questionnaires assessed team-satisfaction with:

- process ("I have performed my tasks efficiently.") - 4 questions

- result ("The aims that I have set have been achieved.") - 4 questions

$\checkmark$ cooperation -4 questions

The software (TAM) related questionnaire included questions related to:

- ease of use ("The software increases my productivity.") -6 questions

- usability ("In total I think the software is useful for my tasks.") - 6 questions

- according to technology acceptance model (TAM) of Davis (1989) model and additionally

- interoperability as a BIM specific feature of software applications 


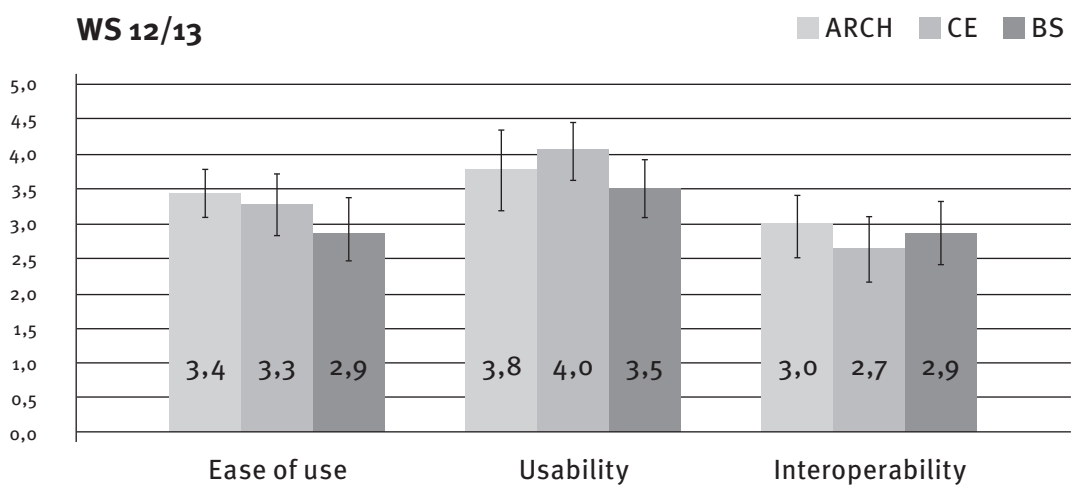

WS $13 / 14$

$\square$ ARCH $\square$ CE $\square$ BS

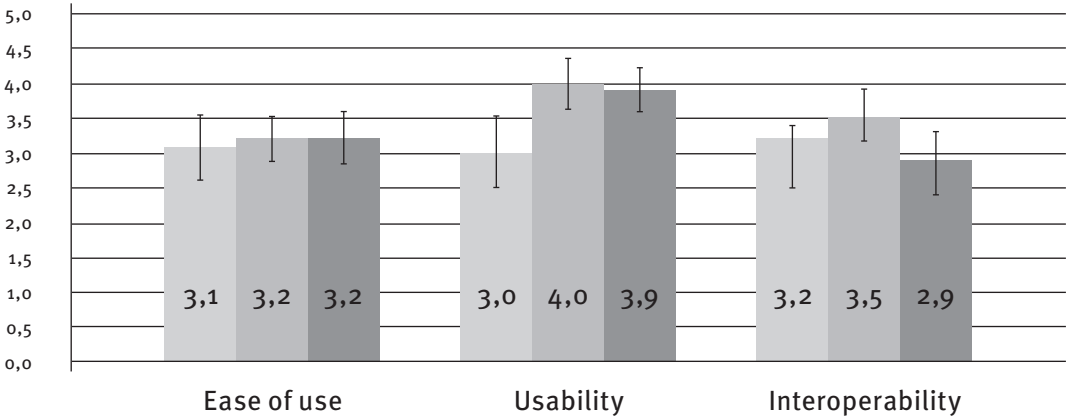

Figure 3: Evaluation of satisfaction with Process, Result and Cooperation for WS 2012/13 and 2013/14

WS $12 / 13$

ARCH CE BS

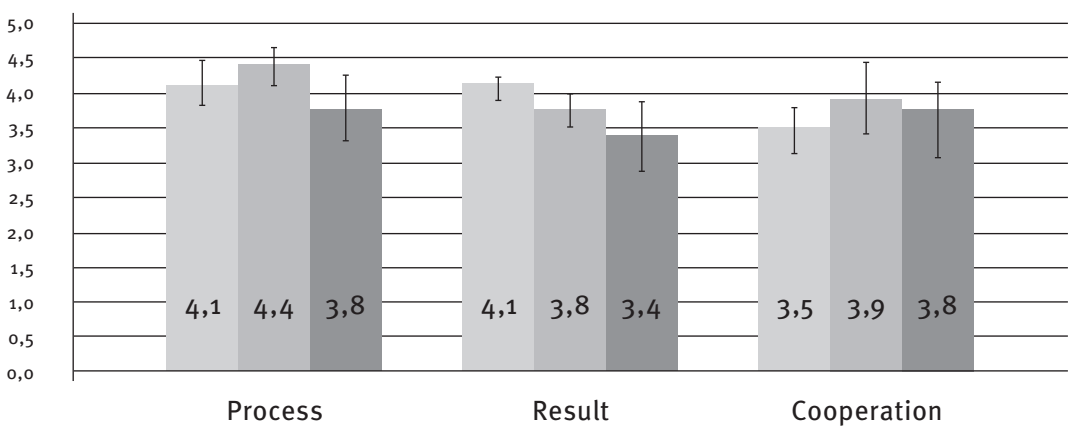

WS $13 / 14$

$\triangle \mathrm{ARCH} \square \mathrm{CE} \square \mathrm{BS}$

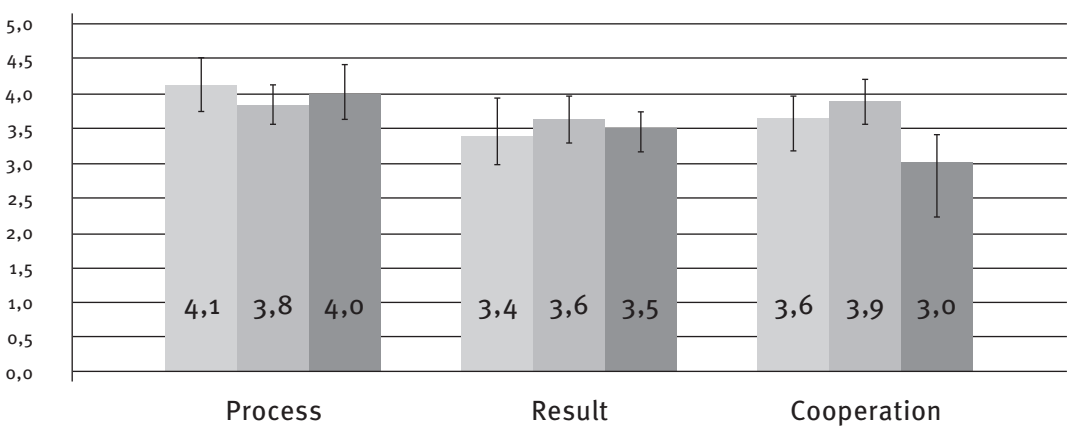

Figure 4: Evaluation of satisfaction with Ease of Use, Usability and Interoperability for WS 2012/13 and 2013/14
The overall results of the questionnaire-evaluation (all the teams, clustered per discipline) for WS $12 / 13$ and WS $13 / 14$ are presented in Fig. 3 for satisfaction and in Fig. 4 for TAM.

By trend the usability is perceived as highest by the civil engineers, since they profit from the models in the process of analysis and simulation. Interoperability is perceived in both cycles as the category with lowest performance, especially so by the subsequent disciplines who import the architectural models. Lack of interoperability is resulting in the wrong interpretation of geometry, leading to significant efforts for repairing of models or even complete re-modelling.

The lowest is satisfaction with the cooperation in the planning team, against the expectation in the second cycle where the kick-off meeting was introduced.

The time-assessment (Fig. 5) shows that the direct communication respective coordination within the team is the second-high time intensive task, next to the actual planning in form of technical planning (modelling) and conceptual design; which supports the hypothesis that BIM-supported planning is communication and coordination intense.

Fig. 6 summarises all of the projects and obtained results per team in the winter semester $13 / 14$. Thereby the used software per team and per discipline is presented, the evaluation of satisfaction and TAM per team, as well as the evaluation of integrated and discipline-specific BIM-models via traffic-light method.

\section{Lessons Learned}

Lessons learned from the pilot-experiment

The hypothesis, that the introduction of BIM alone would support or enhance the integrated planning, was not confirmed. The students were working in sequential manner, start- 


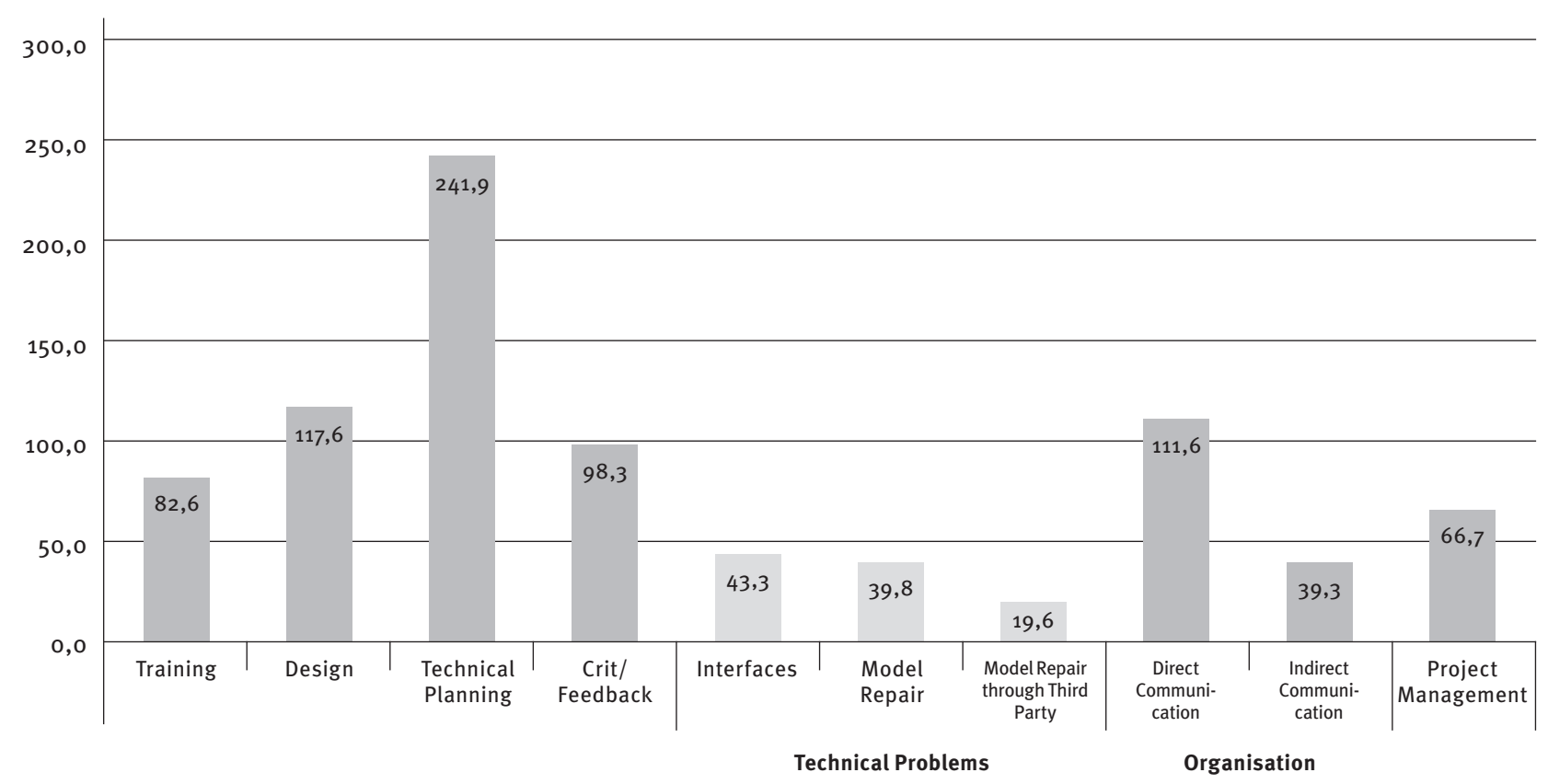

Figure 5: Time effort in hours for specific activity (task), based on evaluation of time sheets in WS 2013/14

ing with the creation and modelling of architectural model, over structural modelling till thermal and daylight simulation and optimization in the end. The teams were not teams, but groups, until the point of last presentation, where the team forming finally took place (the joint model had to be presented). This can be related to the lack of Kick-Off workshop. The participants reported numerous problems and conflict, mostly resulting due to the incompatible software-constellations lacking working interfaces.

Due to the inter-faculty collaboration, the course was awarded varying number of ECTS (credits) at different faculties. The architects obtained in this cycle only 2,0 ECTS, the civil engineers 6,o ECTS and master of building science even 8,0 ECTS in their respective curricula. The difference in the ECTS resulted in numerous conflicts, due to unequal task distribution and related credits / awards.

\section{Lessons learned from}

the second cycle

Learning from the pilot-experiment, the second cycle was more thoroughly structured and designed. Integrated working cycles were interlaced with sequential phases; a Kick-Off Workshop was organized for team-building and -forming. The proposed softwarecombinations were chosen more carefully with respect to interoperability, in order to support the planning process. A better team-spirit, easier coordination and stronger joint vision could be observed as result. In this cycle the awarded ECTS were more balanced, due to the facts that the architects obtained 5,0 ECTS, since BIM course took place as the "Design Studio" in the specific curriculum.

\section{Lessons learned both cycles}

The students have concentrated basically either on the learning of the new software or on the new functionalities of the software (compilation oft he IFC files for data-transfer). The second learning focus was the multidisciplinary collaboration, which is also a learning process. With these simultaneous new tasks, the students were not able to concentrate and optimize the actual design task. This phenomenon was observed in both cycles - this is the so called trade-off of interests, which can be expected when a task with several newly imposed requirements is posed.

Interoperability is one of the most important issues, on the software level, as well as on the level of people and process, being the origin of many conflicts and re-modelling efforts.

Next to the problem of lacking software interoperability, the further cause of conflict was the diverging modelling semantics - the way each discipline perceives and models the space and consequently the geometry.

For example the architectural modelling of the pillar is from slab to slab; where as the structural engineers requires continuous pillar in the full height of built structure for a proper analysis. The structural model obtained from architectural software is full of gaps; automated model repairing procedure is still lacking. The question remains, whose responsibility or task it is to repair the slabs.

The differences considering the modelling quality emerged not only between the students of various disciplines, but also between the teaching team and the students. The teachers criticised the lack of the model and design quality, where as the students 


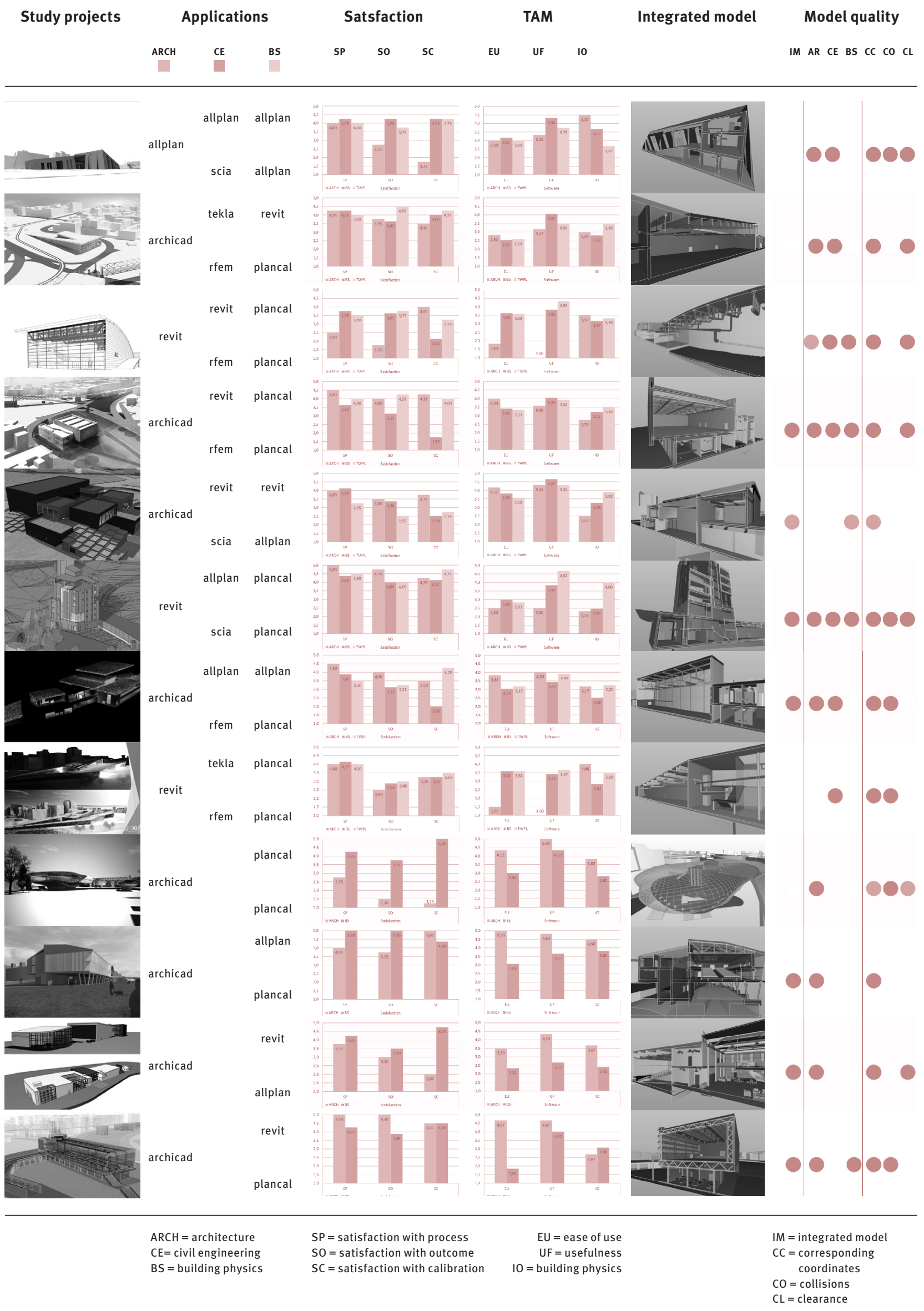


Figure 6: Project Evaluation Summary for WS13/14, Participating Students: Ali H Ahmadalshteihi Amjad, Andersen Jesper Hyllekilde, Brauner Florian, Bucevac Mirjana, Buder Josef, Ciuffi Emanuela, Dobkowska Karolina, Filipczak Dominik, Glodowska Kamila, Gourlis Georgios, Gubina Ekaterina, Hammerschick Michael, Hehenberger Stefan, Ivanova Ira, Kachynska Liubov, Karhan Nikolas, Khelifi Ahmed Anis, Mahmoudzadehvazifeh Ehsan, Malhotra Astrid, Markovic Marija, Mayer Alice, Merentitis Christos,Miremadi Mahtab, Mötz Stefan, Mrowetz Benjamin, Nadianmehr Reza, Oprita Ioana, Padayhag Thommy-James, Pieber Markus, Proskurnina Olga, Rubio Massot Martí, Rusev Miroslav Avramov, Sabanovic Marija, Schachner Moritz, Shadrina Anna, Sharma Atul, Soleimani Babakamali Ronak, Steiner Peter, Tomazovic Milica, Troschl Gerald, Tschida Jacqueline, Vanca Mihai, Varbanov Kosta, Vogl Stefan, Wyrzykowska Aleksandra

evaluated their own performance despite all of the difficulties related to the software and team-communication as good. This can be interpreted as the relative self-assessment - compared to the import-export software problems and necessary team-coordination efforts, the final result for the participants is satisfactory. Thereby employing solemnly the self-assessment through questionnaires and focusgroups for the evaluation of the quality and performance is not sufficient.

The positive experiences related to the collaboration in the integrated processes outweigh the negative ones, especially so for the architects and civil engineers, the data exchange and collaboration in the early design stages in experienced as inspiring and interesting.

However some of the architects feel put under pressure and limited in the creativity when working in such setting. Helpful is the experience in specific discipline, such as e.g. building science master with architectural bachelor background, since the process-knowledge was already there, so the requirements of the discipline could be considered.

Early determination of modelling standards was typical for the very efficient groups. The architect took over management and coordination functions in many groups, since they had free resources after creating the original model. Diverging motivation and incentives can be conflict-triggers, as obvious from the varying ECTS issue, a problem that can be transferred oneto-one to the practice. An intensive collaboration on a joint BIM-knowledge model makes a group-dynamics an important topic in BIM research.

Finally, the organization of a multidisciplinary university course without explicit support and placement in curricula is particularly difficult, since the curricula are very extensive and do not foresee this kind of collaboration. This insight can be used as a parable for the practice, where an awareness for necessary resources and exact planning and design of multidisciplinary collaboration on corporate or project-level is needed.

\section{Conclusion}

The evaluation of the collected data shows that for the realization of integrated planning the sole adoption of BIM-tools is not sufficient. In the pilot experiment the students were planning in sequential manner, the modelling of the one discipline followed in succession to the other. In the second cycle due to the course design (KickOff meeting, a series of joint workshops) it was possible to achieve a better process integration.

The topics of software interoperability dominated the focus group interviews, which served for feedback and reflection, conducted at the end of the course with the partaking disciplines.

The lack of the operability of used software resulted in additional workeffort, leading to conflicts in work-load distribution - e.g. who will undertake the necessary repairs or re-modelling? This problem was especially clear in the so called BIM (Building Information Modelling) to BEM (Building Energy Modelling) step; where architectural models were transferred in the thermal simulation software environment, where the transfer is possible only using proprietary interfaces, and on the other hand numerous geometry interpretation problems emerge due to the semantical model differences.

The students were confronted with two new tasks - learning of the new BIM software or new functionalities related to the data transfer within known BIM software which was not used in the practice; as well as the learning of the simultaneous, interdisciplinary planning. The introduction of two new tasks resulted with relatively low project-quality (design-quality).

The participants anticipated the additional time effort and chose proactively a design of reduced complexity; or were forced to lower the design ambitions; due to the fact that the data transfer and team coordination consumed too much time.

The necessity for further development of open interfaces such as IFC, development of modelling standards and procedures, but also necessity for design of design process; as well as BIM skills and training could be identified as critical factors for successful implementation of BIM in the AEC practice.

Finally we see the hypothesis confirmed that BIM software is useful for planning, simulation and optimization; however these processes require a more intensive communication and team coordination as the design-process using traditional CAD tools. 


\section{Literature}

Azhar, S., et al., 2011. Building information modeling for sustainable design and LEED $®$ rating analysis. Automation in Construction 20, p. 217.

CWIC, 2004. The building technology and construction industry technology roadmap, in "Collaborative Working in Consortium " $\mathrm{A}$. Dawson, Editor. Melbourne.

Davis, F.D., 1989. Perceived usefulness, perceived ease of use, and user acceptance of information technology. MIS Quarterly 13 (3), p. 319.

Fellows, R., Liu, A.M.M., 2012. Managing organizational interfaces in engineering construction projects: addressing fragmentation and boundary issues across multiple interfaces. Construction Management and Economics $30(8)$, p. 653.

Gu, N., London, K., 2010. Understanding and facilitating BIM adoption in the AEC industry. Automation in Construction 19, p. 988.

Jung, Y., Joo, M., 2011. Building information modelling (BIM) framework for practical implementation. Automation in Construction 20, p. 126.

Krueger R.A., Casey, M.A., 2009. Focus groups. A Practical Guide for Applied Research. SAGE Publications Ltd., Los Angeles.

Linderoth, H.C.J., 2010. Understanding adoption and use of BIM as the creation of actor networks. Automation in Construction 19, p. 66.

Mayring, P., 2009. Qualitative Inhaltsanalyse. Grundlagen und Techniken. UTB/Beltz Verlag.

McGraw-Hill Construction, 2010. The Business Value of BIM in Europe, H.M. Bernstein Editor. Smart Market Report.

Penttilä, H., 2006. Describing the changes in architectural information technology to understand design complexity and free-form architectural expression. ITcon 11, Special Issue "The Effects of CAD on Building Form and Design Quality”, p. 395.

Schreier, M., 2012. Qualitative Content Analysis in Practice. SAGE Publications Ltd., London.

Succar, B., 2009. Building information modelling framework: A research and delivery foundation for industry stakeholders. Automation in Construction 18, p. 357. 